\title{
GLOBAL SMOOTH SOLUTIONS OF THE GENERALIZED KS-CGL EQUATIONS FOR FLAMES GOVERNED BY A SEQUENTIAL REACTION*
}

\author{
CHANGHONG GUO ${ }^{\dagger}$, SHAOMEI FANG ${ }^{\ddagger}$, AND BOLING GUO $§$
}

\begin{abstract}
In this paper, we investigate the periodic initial value problem and Cauchy problem of the generalized Kuramoto-Sivashinsky-complex Ginzburg-Landau (GKS-CGL) equations for flames governed by a sequential reaction. We prove the global existence and uniqueness of solutions to these two problems in various spatial dimensions via delicate a priori estimates, the Galerkin method, and so-called continuity method.
\end{abstract}

Key words. Global existence, generalized KS-CGL equations, sequential reaction, a priori estimates, Galerkin method.

AMS subject classifications. 35D35, 35Q56.

\section{Introduction}

In this paper, we consider the global existence and uniqueness of solutions for the following coupled generalized Kuramoto-Sivashinsky-complex Ginzburg-Landau (GKS-CGL) equations for flames:

$$
\begin{gathered}
P_{t}=\xi P+(1+i \mu) \Delta P-(1+i \nu)|P|^{2} P-\nabla P \nabla Q-r_{1} P \Delta Q-g r_{2} P \Delta^{2} Q, \\
Q_{t}=-\Delta Q-g \Delta^{2} Q+\delta \Delta^{3} Q-\frac{1}{2}|\nabla Q|^{2}-\eta|P|^{2}
\end{gathered}
$$

with the periodic initial conditions

$$
\begin{gathered}
P\left(x+L e_{i}, t\right)=P(x, t), Q\left(x+L e_{i}, t\right)=Q(x, t), x \in \Omega, t \geq 0, \\
P(x, 0)=P_{0}(x), \quad Q(x, 0)=Q_{0}(x), \quad x \in \Omega,
\end{gathered}
$$

or the initial conditions

$$
P(x, 0)=P_{0}(x), \quad Q(x, 0)=Q_{0}(x), \quad x \in \mathbb{R}^{n} .
$$

The spatial domain $\Omega$ is a bounded domain in $n$-dimensional real Euclidean space $\mathbb{R}^{n}(n=1,2)$, and the time $t \geq 0$. The complex function $P(x, t)$ is the rescaled amplitude of the flame oscillations, the real function $Q(x, t)$ is the deformation of the first front, and both of them are $L$-periodic. The coefficient $\xi= \pm 1$. The Landau coefficients $\mu, \nu$ and the coupling coefficient $\eta>0$ are real, while the parameters $r_{1}$ and $r_{2}$ are complex, $r_{1}=r_{1 r}+i r_{1 i}, r_{2}=r_{2 r}+i r_{2 i}$. The coefficient $g>0$ is proportional to the

\footnotetext{
${ }^{*}$ Received: February 8, 2013; accepted (in revised form): October 28, 2013. Communicated by Jack Xin.

This work was supported by the National Natural Science Foundation of China (No. 11271141).

${ }^{\dagger}$ School of Management, Guangdong University of Technology, Guangzhou 510520, P.R. China (Guo02031894285@163.com).

${ }^{\ddagger}$ Corresponding author. Department of Mathematics, South China Agricultural University, Guangzhou 510640, P.R. China (dz90@scau.edu.cn).

$\S$ Institute of Applied Physics and Computational Mathematics, P.O.Box 8009, Beijing 100088, P.R. China (gbl@iapcm.ac.cn).
} 
supercriticality of the oscillatory mode. $\delta>0$ is a constant, $L>0$ is the period, and $e_{i}$ is the standard coordinate vector.

If we take $\delta=0$ in equation (1.2), the coupled GKS-CGL equations (1.1) (1.2) are reduced to the classical KS-CGL equations [1], which describe the nonlinear interaction between the monotonic and oscillatory modes of instability of the two uniformly propagating flame fronts in a sequential reaction. Specifically, they describe both the long-wave evolution of the oscillatory mode near the oscillatory instability threshold, and the evolution of the monotonic mode. For the background of the uniformly propagating premixed flame fronts and the derivation of the KS-CGL model, we refer to $[1,2,3,4]$ for details. If there were no coupling with the monotonic mode (terms with $Q$ in equation (1.1)), then equation (1.1) would be the well-known CGL equation that usually describes the weakly nonlinear evolution of a long-scale instability [5]. If we take $\delta=0$ and the coupling coefficient $\eta=0$ in equation (1.2), then equation (1.2) reduces to the well-known KS equation [6], which governs the flame front's spatiotemporal evolution and describes stationary (time independent) spatially periodic patterns as well as further transitions. As can be seen, the coupled GKS-CGL equations (1.1) (1.2) can better describe the dynamical behavior for flames governed by a sequential reaction, because they generalize the KS equations, the CGL equations, or the KS-CGL equations.

So far, the mathematical analysis and physical study about the CGL equation and KS equation have been done by many researchers. For example, the existence of global solutions and attractor for the CGL equation and KS equation are studied in $[7,8,9,10,11]$. For some other results, see $[12,13,14,15]$ and references therein. However, little progress has been obtained for the coupled KS-CGL equations because they were derived to describe the nonlinear evolution for flames by A. A. Golovin, et al. [1]. They studied the traveling waves of the coupled equations numerically and continued to study the spiral waves [16], which exhibit new types of instabilities. Meanwhile, there are few works which consider mathematical analytical properties of the KS-CGL equations and generalized KS-CGL equations, even the existence and uniqueness of the solutions. In this paper, we are concerned with the global existence and uniqueness theory for the periodic initial value problem (1.1)-(1.4) and the Cauchy problem $(1.1)(1.2)(1.5)$ via delicate a priori estimates and the Galerkin method. For the Cauchy problem $(1.1)(1.2)(1.5)$, we suppose that $P(x, t), Q(x, t)$, and some of their derivatives with respect to $x$ tend to zero as $|x| \rightarrow \infty$.

The rest of paper is organized as follows. In Section 2, we briefly give some notations and preliminaries. In Section 3, we will establish a priori estimates for the solutions of the periodic initial value problem (1.1)-(1.4). In Section 4, the existence and uniqueness of the global smooth solutions for the periodic initial value problem (1.1)-(1.4) are obtained via the Galerkin method and so-called continuity method. In Section 5, we employ the usual method of limiting process to obtain the solutions for the Cauchy problem (1.1)(1.2)(1.5).

\section{Notations and preliminaries}

We shall use the following conventional notations throughout the paper. Let $L_{p e r}^{k}$ and $H_{p e r}^{k}, k=1,2, \cdots$ denote the Hilbert and Sobolev spaces of $L$-periodic, complexvalued functions endowed with the usual $L^{2}$ inner product $(u, v)=\int_{\Omega} u(x) \bar{v}(x) d x$ and 
norms

$$
\|u\|_{L^{2}}=\sqrt{(u, u)}, \quad\|u\|_{H^{k}}=\left(\sum_{|\alpha| \leq k}\left\|D^{\alpha} u(x)\right\|\right)^{\frac{1}{2}} .
$$

Here $\bar{v}$ denotes the complex conjugate of $v$. For brevity, we write $\|u\|=\|u\|_{L^{2}}$ and denote the $L^{p}$-norm by $\|u\|_{p}=\left(\int_{\Omega}|u|^{p} d x\right)^{1 / p}$. Without any ambiguity, we denote a generic positive constant by $C$ which may vary from line to line.

In the following sections, we frequently use following inequalities.

LEMma 2.1 (Young's inequality with $\varepsilon[17]$ ). Let $a>0, b>0,1<p, q<\infty, \frac{1}{p}+\frac{1}{q}=$ 1. Then

$$
a b \leq \varepsilon a^{p}+C(\varepsilon) b^{q},
$$

for $C(\varepsilon)=(\varepsilon p)^{-q / p} q^{-1}$.

LEMMA 2.2 (Gagliardo-Nirenberg inequality [18]). Let $\Omega$ be a bounded domain with $\partial \Omega$ in $C^{m}$, and let $u$ be any function in $W^{m, r}(\Omega) \cap L^{q}(\Omega), 1 \leq q, r \leq \infty$. For any integer $j, 0 \leq j<m$, and for any number a in the interval $j / m \leq a \leq 1$, set

$$
\frac{1}{p}=\frac{j}{n}+a\left(\frac{1}{r}-\frac{m}{n}\right)+(1-a) \frac{1}{q} .
$$

If $m-j-n / r$ is not a nonnegative integer, then

$$
\left\|D^{j} u\right\|_{L^{p}} \leq C\|u\|_{W^{m, r}}^{a}\|u\|_{L^{q}}^{1-a} .
$$

If $m-j-n / r$ is a nonnegative integer, then (2.1) holds for $a=j / m$. The constant $C$ depends only on $\Omega, r, q, j$, and $a$.

In the sequel, we will use the following inequalities as the specific cases of the Gagliardo-Nirenberg inequality:

$$
\begin{gathered}
\left\|D^{j} u\right\|_{L^{\infty}} \leq C\|u\|_{H^{m}}^{a}\|u\|^{1-a}, \quad m a=j+n / 2, \\
\left\|D^{j} u\right\|_{L^{2}} \leq C\|u\|_{H^{m}}^{a}\|u\|^{1-a}, \quad m a=j, \\
\left\|D^{j} u\right\|_{L^{4}} \leq C\|u\|_{H^{m}}^{a}\|u\|^{1-a}, \quad m a=j+n / 4 .
\end{gathered}
$$

\section{A priori estimates}

In this section, we derive some a priori estimates for the solutions of the problem (1.1)-(1.4). Firstly we have the following result.

Lemma 3.1. Assume $P_{0}(x) \in L_{\text {per }}^{2}(\Omega), Q_{0}(x) \in H_{p e r}^{1}(\Omega)$, and suppose that $2 \delta-g^{2} r_{2 r}^{2}>$ 0. Then for the solutions of the problem (1.1)-(1.4), we have

$$
\|P\|^{2} \leq e^{K_{1} t}\left(\left\|P_{0}\right\|^{2}+\left\|\nabla Q_{0}\right\|^{2}\right), \quad\|\nabla Q\|^{2} \leq e^{K_{1} t}\left(\left\|P_{0}\right\|^{2}+\left\|\nabla Q_{0}\right\|^{2}\right),
$$

where $K_{1}$ is a positive constant.

Proof. First we differentiate equation (1.2) with respect to $x$ once and set

$$
W=\nabla Q .
$$


Then equation (1.1) and (1.2) can be rewritten as

$$
\begin{gathered}
P_{t}=\xi P+(1+i \mu) \Delta P-(1+i \nu)|P|^{2} P-\nabla P W-r_{1} P \nabla W-g r_{2} P \nabla \Delta W, \\
W_{t}=-\Delta W-g \Delta^{2} W+\delta \Delta^{3} W-W \nabla W-\eta \nabla\left(|P|^{2}\right) .
\end{gathered}
$$

Multiplying (3.3) by $\bar{P}$, integrating with respect to $x$ over $\Omega$, and taking the real part, we obtain

$$
\begin{array}{r}
\frac{1}{2} \frac{d}{d t}\|P\|^{2}=\xi\|P\|^{2}-\|\nabla P\|^{2}-\int_{\Omega}|P|^{4} d x-\operatorname{Re} \int_{\Omega} \nabla P \bar{P} W d x \\
-r_{1 r} \int_{\Omega}|P|^{2} \nabla W d x-g r_{2 r} \int_{\Omega}|P|^{2} \nabla \Delta W d x,
\end{array}
$$

where

$$
-\operatorname{Re} \int_{\Omega} \nabla P \bar{P} W d x=\frac{1}{2} \int_{\Omega}|P|^{2} \nabla W d x .
$$

On the other hand, multiplying (3.4) by $W$ and integrating over $\Omega$, we have

$$
\frac{1}{2} \frac{d}{d t}\|W\|^{2}=\|\nabla W\|^{2}-g\|\Delta W\|^{2}-\delta\|\nabla \Delta W\|^{2}-\int_{\Omega} W^{2} \nabla W d x-\eta \int_{\Omega} \nabla\left(|P|^{2}\right) W d x,
$$

where

$$
\int_{\Omega} W^{2} \nabla W d x=\sum_{i=1}^{n} \int_{\Omega} \frac{\partial W}{\partial x_{i}} W^{2} d x=\frac{1}{3} \sum_{i=1}^{n} \int_{\Omega} \frac{\partial}{\partial x_{i}}\left(W^{3}\right) d x=0
$$

and

$$
-\eta \int_{\Omega} \nabla\left(|P|^{2}\right) W d x=\eta \int_{\Omega}|P|^{2} \nabla W d x
$$

Adding (3.5) and (3.7) together, and noticing (3.6), (3.8), and (3.9), there holds

$$
\begin{aligned}
& \frac{d}{d t}\left(\|P\|^{2}+\|W\|^{2}\right) \\
= & 2 \xi\|P\|^{2}-2\|\nabla P\|^{2}-2 \int_{\Omega}|P|^{4} d x+2\|\nabla W\|^{2}-2 g\|\Delta W\|^{2} \\
& \quad-2 \delta\|\nabla \Delta W\|^{2}+\left(1+2 \eta-2 r_{1 r}\right) \int_{\Omega}|P|^{2} \nabla W d x-2 g r_{2 r} \int_{\Omega}|P|^{2} \nabla \Delta W d x .
\end{aligned}
$$

According to the Gagliardo-Nirenberg inequality (2.3), we have

$$
\begin{aligned}
& 2\|\nabla W\|^{2}+\left(1+2 \eta-2 r_{1 r}\right) \int_{\Omega}|P|^{2} \nabla W d x \\
\leq & 2\|\nabla W\|^{2}+\left|1+2 \eta-2 r_{1 r}\right|\left(\int_{\Omega}|P|^{4} d x\right)^{\frac{1}{2}}\|\nabla W\| \\
\leq & \varepsilon_{1} \int_{\Omega}|P|^{4} d x+C\|\nabla W\|^{2} \\
\leq & \varepsilon_{1} \int_{\Omega}|P|^{4} d x+C\|W\|_{H^{3}}^{\frac{2}{3}}\|W\|^{\frac{4}{3}}
\end{aligned}
$$




$$
\leq \varepsilon_{1} \int_{\Omega}|P|^{4} d x+\frac{\varepsilon_{2}}{2}\|\nabla \Delta W\|^{2}+C_{1}\|W\|^{2},
$$

and

$$
\left.\left.\left|-2 g r_{2 r} \int_{\Omega}\right| P\right|^{2} \nabla \Delta W d x\left|\leq \frac{\varepsilon_{2}}{2}\|\nabla \Delta W\|^{2}+\frac{2 g^{2} r_{2 r}^{2}}{\varepsilon_{2}} \int_{\Omega}\right| P\right|^{4} d x .
$$

Combining (3.10)-(3.12) and noticing that $|\xi|=1$, we have

$$
\begin{aligned}
\frac{d}{d t}\left(\|P\|^{2}+\|W\|^{2}\right) \leq 2 \| & P\left\|^{2}+C_{1}\right\| W\left\|^{2}-2\right\| \nabla P\left\|^{2}-2 g\right\| \Delta W \|^{2} \\
& -\left(2 \delta-\varepsilon_{2}\right)\|\nabla \Delta W\|^{2}-\left(2-\frac{2 g^{2} r_{2 r}^{2}}{\varepsilon_{2}}-\varepsilon_{1}\right) \int_{\Omega}|P|^{4} d x .
\end{aligned}
$$

Under the condition $2 \delta-g^{2} r_{2 r}^{2}>0$, we can choose $\varepsilon_{2}$ such that $0<g^{2} r_{2 r}^{2}<\varepsilon_{2} \leq 2 \delta$ and choose $\varepsilon_{1}$ to be sufficiently small such that $2-\frac{2 g^{2} r_{2 r}^{2}}{\varepsilon_{2}}-\varepsilon_{1}>0$. Then we have

$$
\begin{aligned}
& \frac{d}{d t}\left(\|P\|^{2}+\|W\|^{2}\right)+2\|\nabla P\|^{2}+2 g\|\Delta W\|^{2} \\
& +\left(2 \delta-\varepsilon_{2}\right)\|\nabla \Delta W\|^{2}+\left(2-\frac{2 g^{2} r_{2 r}^{2}}{\varepsilon_{2}}-\varepsilon_{1}\right) \int_{\Omega}|P|^{4} d x . \\
\leq & 2\|P\|^{2}+C_{1}\|W\|^{2} \\
\leq & K_{1}\left(\|P\|^{2}+\|W\|^{2}\right),
\end{aligned}
$$

where $K_{1}=\max \left(2, C_{1}\right)$. By Gronwall's inequality, we have

$$
\|P\|^{2}+\|W\|^{2} \leq e^{K_{1} t}\left(\left\|P_{0}\right\|^{2}+\left\|W_{0}\right\|^{2}\right),
$$

where $K_{1}$ is a positive constant. Combining the transformation (3.2), one can complete the proof of Lemma 3.1.

Lemma 3.2. Assume $P_{0}(x) \in H_{p e r}^{1}(\Omega), Q_{0}(x) \in H_{p e r}^{3}(\Omega)$, and $2 \delta-g^{2} r_{2 r}^{2}>0$. If $n=2$, we also suppose $|\nu| \leq \sqrt{3}$. Then for the solutions of the problem (1.1)-(1.4), we have

$$
\begin{gathered}
\|\nabla P\|^{2} \leq e^{K_{2} t}\left(\left\|\nabla P_{0}\right\|^{2}+\left\|\Delta Q_{0}\right\|^{2}+\left\|\nabla \Delta Q_{0}\right\|^{2}+C t\right), \\
\|\Delta Q\|^{2}+\|\nabla \Delta Q\|^{2} \leq e^{K_{2} t}\left(\left\|\nabla P_{0}\right\|^{2}+\left\|\Delta Q_{0}\right\|^{2}+\left\|\nabla \Delta Q_{0}\right\|^{2}+C t\right),
\end{gathered}
$$

where $K_{2}$ and $C$ are positive constants.

Proof. Similar to the first step in Lemma 3.1, we use the transformed equations. Multiplying $(3.3)$ by $(-\Delta \bar{P})$, integrating with respect to $x$ over $\Omega$, and taking the real part, we get

$$
\begin{aligned}
\frac{1}{2} \frac{d}{d t}\|\nabla P\|^{2}=\xi \| & \nabla P\left\|^{2}-\right\| \Delta P \|^{2}+\operatorname{Re} \int_{\Omega}(1+i \nu)|P|^{2} P \Delta \bar{P} d x \\
& +\operatorname{Re} \int_{\Omega} \nabla P \Delta \bar{P} W d x+\operatorname{Re} \int_{\Omega} r_{1} P \Delta \bar{P} \nabla W d x+\operatorname{Re} \int_{\Omega} g r_{2} P \Delta \bar{P} \nabla \Delta W d x .
\end{aligned}
$$


Taking the inner product of (3.4) with $(-\Delta W)$ and $\Delta^{2} W$ over $\Omega$ respectively,

$$
\begin{aligned}
\frac{1}{2} \frac{d}{d t}\|\nabla W\|^{2}=\|\Delta W\|^{2}-g\|\nabla \Delta W\|^{2}-\delta\left\|\Delta^{2} W\right\|^{2} \\
+\int_{\Omega} W \nabla W \Delta W d x+\eta \int_{\Omega} \nabla\left(|P|^{2}\right) \Delta W d x
\end{aligned}
$$

and

$$
\begin{aligned}
\frac{1}{2} \frac{d}{d t}\|\Delta W\|^{2}= & \|\nabla \Delta W\|^{2}-g\left\|\Delta^{2} W\right\|^{2}-\delta\left\|\nabla \Delta^{2} W\right\|^{2} \\
& -\int_{\Omega} W \nabla W \Delta^{2} W d x-\eta \int_{\Omega} \nabla\left(|P|^{2}\right) \Delta^{2} W d x .
\end{aligned}
$$

Adding (3.18), (3.19), and (3.20) together yields that

$$
\begin{aligned}
& \frac{d}{d t}\left(\|\nabla P\|^{2}+\|\nabla W\|^{2}+\|\Delta W\|^{2}\right) \\
=2 \xi \| & \nabla P\left\|^{2}-2\right\| \Delta P\left\|^{2}+2\right\| \Delta W\left\|^{2}-2(g-1)\right\| \nabla \Delta W \|^{2} \\
& -2(\delta+g)\left\|\Delta^{2} W\right\|^{2}-2 \delta\left\|\nabla \Delta^{2} W\right\|^{2} \\
& +2 \operatorname{Re} \int_{\Omega}(1+i \nu)|P|^{2} P \Delta \bar{P} d x+2 \operatorname{Re} \int_{\Omega} \nabla P \Delta \bar{P} W d x \\
& +2 \operatorname{Re} \int_{\Omega} r_{1} P \Delta \bar{P} \nabla W d x+2 \operatorname{Re} \int_{\Omega} g r_{2} P \Delta \bar{P} \nabla \Delta W d x \\
& +2 \int_{\Omega} W \nabla W \Delta W d x+2 \eta \int_{\Omega} \nabla\left(|P|^{2}\right) \Delta W d x \\
& -2 \int_{\Omega} W \nabla W \Delta^{2} W d x-2 \eta \int_{\Omega} \nabla\left(|P|^{2}\right) \Delta^{2} W d x .
\end{aligned}
$$

Now we need to majorize the right hand side of (3.21). Notice that when the spatial dimension is $n=1$, it is easy to find that

$$
\begin{aligned}
\left.\left|2 \operatorname{Re} \int_{\Omega}(1+i \nu)\right| P\right|^{2} P \bar{P}_{x x} d x \mid & \leq 2|1+i \nu|\|P\|_{L^{8}}^{2}\|P\|_{L^{4}}\left\|P_{x x}\right\| \\
& \leq C\left\|P_{x x}\right\|^{\frac{3}{8}}\|P\|^{\frac{13}{8}}\left\|P_{x x}\right\|^{\frac{1}{8}}\|P\|^{\frac{7}{8}}\left\|P_{x x}\right\| \\
& \leq \frac{1}{2}\left\|P_{x x}\right\|^{2}+C\|P\|^{10} \\
& \leq \frac{1}{2}\left\|P_{x x}\right\|^{2}+C .
\end{aligned}
$$

While $n=2$, we handle this term as follows:

$$
\begin{aligned}
& 2 \operatorname{Re} \int_{\Omega}(1+i \nu)|P|^{2} P \Delta \bar{P} d x \\
= & -2 \operatorname{Re} \int_{\Omega}(1+i \nu)\left(|P|^{2}|\nabla P|^{2}+P \nabla \bar{P} \nabla\left(|P|^{2}\right)\right) d x \\
= & -2 \int_{\Omega}|P|^{2}|\nabla P|^{2} d x-\int_{\Omega}\left|\nabla\left(|P|^{2}\right)\right|^{2} d x+\nu \int_{\Omega} \nabla\left(|P|^{2} \mid\right) \cdot i(\bar{P} \nabla P-P \nabla \bar{P}) d x \\
= & -\frac{1}{2} \int_{\Omega}\left(3\left|\nabla\left(|P|^{2}\right)\right|^{2}-2 \nu \nabla\left(|P|^{2}\right) \cdot i(\bar{P} \nabla P-P \nabla \bar{P})+|\bar{P} \nabla P-P \nabla \bar{P}|^{2}\right) d x .
\end{aligned}
$$


We observe that the integrand in the last term in (3.23) is a quadratic form, and under the condition of $|\nu| \leq \sqrt{3}$, the matrix $\left(\begin{array}{cc}3 & -\nu \\ -\nu & 1\end{array}\right)$ is nonnegative definite, which implies the integrand is nonnegative. Thus we have

$$
2 \operatorname{Re} \int_{\Omega}(1+i \nu)|P|^{2} P \Delta \bar{P} d x<0 \leq \frac{1}{2}\|\Delta P\|^{2} .
$$

Meanwhile, according to Young's inequality with $\varepsilon$ and Gagliardo-Nirenberg inequality (2.2), (2.3), and (2.4), we obtain the following estimates:

$$
\begin{aligned}
& 2\|\Delta W\|^{2}-2(g-1)\|\nabla \Delta W\|^{2}+\left|2 \operatorname{Re} \int_{\Omega} \nabla P \Delta \bar{P} W d x\right| \\
& +\left|2 \operatorname{Re} \int_{\Omega} r_{1} P \Delta \bar{P} \nabla W d x\right|+\left|2 \operatorname{Re} \int_{\Omega} g r_{2} P \Delta \bar{P} \nabla \Delta W d x\right|+\left|2 \int_{\Omega} W \nabla W \Delta W d x\right| \\
& \leq 2\|\Delta W\|^{2}+2|g-1|\|\nabla \Delta W\|^{2}+2\|W\|_{\infty}\|\nabla P\|\|\Delta P\| \\
& +2\left|r_{1}\right|\|P\|\|\Delta P\|\|\nabla W\|_{\infty}+2 g\left|r_{2}\right|\|P\|\|\Delta P\|\|\nabla \Delta W\|_{\infty}+2\|W\|\|\nabla W\|_{\infty}\|\Delta W\| \\
& \leq 2\|\Delta W\|^{2}+2|g-1|\|\nabla \Delta W\|^{2}+C\|W\|_{H^{4}}^{\frac{n}{8}}\|W\|^{\frac{8-n}{8}}\|P\|_{H^{2}}^{\frac{1}{2}}\|P\|^{\frac{1}{2}}\|\Delta P\| \\
& +\frac{1}{6}\|\Delta P\|^{2}+C\|W\|_{H^{3}}^{\frac{2+n}{3}}\|W\|^{\frac{4-n}{3}}+\frac{1}{6}\|\Delta P\|^{2} \\
& +C\|\nabla \Delta W\|_{\infty}^{2}+C\|W\|_{H^{4}}^{\frac{2+n}{8}}\|W\|^{\frac{6-n}{8}}\|\Delta W\| \\
& \leq 2\|\Delta W\|^{2}+C\|W\|_{H^{4}}^{\frac{3}{2}}\|W\|^{\frac{1}{2}}+C\|W\|_{H^{4}}^{\frac{n}{8}}\|W\|^{\frac{8-n}{8}}\|P\|_{H^{2}}^{\frac{1}{2}}\|P\|^{\frac{1}{2}}\|\Delta P\| \\
& +\frac{1}{6}\|\Delta P\|^{2}+C\|W\|_{H^{3}}^{\frac{2+n}{3}}\|W\|^{\frac{4-n}{3}}+\frac{1}{6}\|\Delta P\|^{2}+\frac{1}{6}\|\Delta P\|^{2} \\
& +C\|W\|_{H^{5}}^{\frac{6+n}{5}}\|W\|^{\frac{4-n}{5}}+C\|W\|_{H^{4}}^{\frac{2+n}{8}}\|W\|^{\frac{6-n}{8}}\|\Delta W\| \\
& \leq \frac{1}{2}\|\Delta P\|^{2}+\frac{\delta+g}{2}\left\|\Delta^{2} W\right\|^{2}+\frac{\delta}{2}\left\|\nabla \Delta^{2} W\right\|^{2}+C_{2}\|\nabla P\|^{2}+C_{3}\|\nabla W\|^{2}+C_{4}\|\Delta W\|^{2}+C .
\end{aligned}
$$

After a similar computation we also get

$$
\begin{aligned}
\left|2 \eta \int_{\Omega} \nabla\left(|P|^{2}\right) \Delta W d x\right| & =\left.\left|2 \eta \int_{\Omega}\right| P\right|^{2} \nabla \Delta W d x \mid \\
& \leq 2 \eta\|\nabla \Delta W\|\|P\|_{L^{4}}^{2} \\
& \leq C\|W\|_{H^{4}}^{\frac{3}{2}}\|W\|^{\frac{1}{2}}\|P\|_{H^{1}}^{\frac{n}{2}}\|P\|^{\frac{4-n}{2}} \\
& \leq \frac{\delta+g}{2}\left\|\Delta^{2} W\right\|^{2}+C_{5}\|\nabla P\|^{2}+C_{6}\|\nabla W\|^{2}+C_{7}\|\Delta W\|^{2}+C,
\end{aligned}
$$

and

$$
\begin{aligned}
& \left|-2 \int_{\Omega} W \nabla W \Delta^{2} W d x-2 \eta \int_{\Omega} \nabla\left(|P|^{2}\right) \Delta^{2} W d x\right| \\
\leq & \left|\int_{\Omega} W^{2} \nabla \Delta^{2} W d x\right|+\left.2 \eta\left|\int_{\Omega}\right| P\right|^{2} \nabla \Delta^{2} W d x \mid \\
\leq & \frac{\delta}{2}\left\|\nabla \Delta^{2} W\right\|^{2}+C\left(\|W\|_{L^{4}}^{4}+\|P\|_{L^{4}}^{4}\right) \\
\leq & \frac{\delta}{2}\left\|\nabla \Delta^{2} W\right\|^{2}+C\left(\|W\|_{H^{1}}^{n}\|W\|^{4-n}+\|P\|_{H^{1}}^{n}\|P\|^{4-n}\right)
\end{aligned}
$$




$$
\leq \frac{\delta}{2}\left\|\nabla \Delta^{2} W\right\|^{2}+C_{8}\|\nabla P\|^{2}+C_{9}\|\nabla W\|^{2}+C .
$$

Substituting (3.22)-(3.27) into (3.21) and noticing that $|\xi|=1$, we have

$$
\begin{aligned}
& \frac{d}{d t}\left(\|\nabla P\|^{2}+\|\nabla W\|^{2}+\|\Delta W\|^{2}\right)+\|\Delta P\|^{2}+(\delta+g)\left\|\Delta^{2} W\right\|^{2}+\delta\left\|\nabla \Delta^{2} W\right\|^{2} \\
\leq & \left(2+C_{2}+C_{5}+C_{8}\right)\|\nabla P\|^{2}+\left(C_{3}+C_{6}+C_{9}\right)\|\nabla W\|^{2}+\left(C_{4}+C_{7}\right)\|\Delta W\|^{2}+C \\
\leq & K_{2}\left(\|\nabla P\|^{2}+\|\nabla W\|^{2}+\|\Delta W\|^{2}\right)+C,
\end{aligned}
$$

where $K_{2}=\max \left(2+C_{2}+C_{5}+C_{8}, C_{3}+C_{6}+C_{9}, C_{4}+C_{7}\right)$ is a positive constant. Applying Gronwall's inequality, we have

$$
\|\nabla P\|^{2}+\|\nabla W\|^{2}+\|\Delta W\|^{2} \leq e^{K_{2} t}\left(\left\|\nabla P_{0}\right\|^{2}+\left\|\nabla W_{0}\right\|^{2}+\left\|\Delta W_{0}\right\|^{2}+C t\right) .
$$

This completes the proof of Lemma 3.2 with the transformation (3.2).

Corollary 3.3. Under the conditions of Lemma 3.2, we have the estimates

$$
\|P\|_{H_{p e r}^{1}} \leq C, \quad\|\nabla Q\|_{\infty} \leq C,
$$

where $C$ is a positive constant.

Proof. From Lemma 3.1, Lemma 3.2, and the Gagliardo-Nirenberg inequality (2.2), we have $\|\nabla Q\|_{\infty} \leq C\|\nabla Q\|_{H^{2}}^{\frac{n}{4}}\|\nabla Q\|^{\frac{4-n}{4}} \leq C$, which concludes (3.30).

Lemma 3.4. Assume that $P_{0}(x) \in H_{p e r}^{2}(\Omega), Q_{0}(x) \in H_{p e r}^{4}(\Omega)$, and the conditions of Lemma 3.2. Then for the solutions of the problem (1.1)-(1.4), we have the estimates

$$
\|\Delta P\|^{2}+\left\|\Delta^{2} Q\right\|^{2} \leq e^{K_{3} t}\left(\left\|\Delta P_{0}\right\|^{2}+\left\|\Delta^{2} Q_{0}\right\|^{2}+C t\right),
$$

where $K_{3}$ and $C$ are positive constants.

Proof. $\quad$ After making the transformation (3.2), we take the inner product of (3.3) with $\Delta^{2} \bar{P}$ over $\Omega$ and take the real part, and obtain

$$
\begin{aligned}
& \frac{1}{2} \frac{d}{d t}\|\Delta P\|^{2} \\
= & \xi\|\Delta P\|^{2}-\|\nabla \Delta P\|^{2}-\operatorname{Re} \int_{\Omega}(1+i \nu)|P|^{2} P \Delta^{2} \bar{P} d x \\
& \quad-\operatorname{Re} \int_{\Omega} \nabla P \Delta^{2} \bar{P} W d x-\operatorname{Re} \int_{\Omega} r_{1} P \Delta^{2} \bar{P} \nabla W d x-\operatorname{Re} \int_{\Omega} g r_{2} P \Delta^{2} \bar{P} \nabla \Delta W d x .
\end{aligned}
$$

Multiplying (3.4) by $\left(-\Delta^{3} W\right)$ and integrating with respect to $x$ over $\Omega$,

$$
\begin{aligned}
\frac{1}{2} \frac{d}{d t}\|\nabla \Delta W\|^{2}=\left\|\Delta^{2} W\right\|^{2}-g\left\|\nabla \Delta^{2} W\right\|^{2}-\delta\left\|\Delta^{3} W\right\|^{2} \\
\\
\quad+\int_{\Omega} W \nabla W \Delta^{3} W d x+\eta \int_{\Omega} \nabla\left(|P|^{2}\right) \Delta^{3} W d x .
\end{aligned}
$$

Adding the above two equalities yields

$$
\begin{aligned}
& \frac{d}{d t}\left(\|\Delta P\|^{2}+\|\nabla \Delta W\|^{2}\right) \\
= & 2 \xi\|\Delta P\|^{2}-2\|\nabla \Delta P\|^{2}+2\left\|\Delta^{2} W\right\|^{2}-2 g\left\|\nabla \Delta^{2} W\right\|^{2}-2 \delta\left\|\Delta^{3} W\right\|^{2}
\end{aligned}
$$




$$
\begin{aligned}
& -2 \operatorname{Re} \int_{\Omega}(1+i \nu)|P|^{2} P \Delta^{2} \bar{P} d x-2 \operatorname{Re} \int_{\Omega} \nabla P \Delta^{2} \bar{P} W d x \\
& -2 \operatorname{Re} \int_{\Omega} r_{1} P \Delta^{2} \bar{P} \nabla W d x-2 \operatorname{Re} \int_{\Omega} g r_{2} P \Delta^{2} \bar{P} \nabla \Delta W d x \\
& +2 \int_{\Omega} W \nabla W \Delta^{3} W d x+2 \eta \int_{\Omega} \nabla\left(|P|^{2}\right) \Delta^{3} W d x
\end{aligned}
$$

First, according to the Gagliardo-Nirenberg inequality, Lemma 3.1, and Lemma 3.2, we have

$$
\begin{aligned}
& \left.\left|-2 \operatorname{Re} \int_{\Omega}(1+i \nu)\right| P\right|^{2} P \Delta^{2} \bar{P} d x \mid \\
= & \left|2 \operatorname{Re} \int_{\Omega}(1+i \nu)\left(|P|^{2} \nabla P \nabla \Delta \bar{P}+(P \nabla \bar{P}+\bar{P} \nabla P) P \nabla \Delta \bar{P}\right) d x\right| \\
\leq & 6|1+i \nu|\|P\|_{\infty}^{2}\|\nabla P\|\|\nabla \Delta P\| \\
\leq & \frac{1}{3}\|\nabla \Delta P\|^{2}+C\|P\|_{H^{2}}^{n}\|P\|^{4-n}\|\nabla P\|^{2} \\
\leq & \frac{1}{3}\|\nabla \Delta P\|^{2}+C_{10}\|\Delta P\|^{2}+C,
\end{aligned}
$$

and

$$
\begin{aligned}
\left|-2 \operatorname{Re} \int_{\Omega} \nabla P \Delta^{2} \bar{P} W d x\right| & =\left|2 \operatorname{Re} \int_{\Omega} \nabla P \nabla \Delta \bar{P} \nabla W d x+2 \operatorname{Re} \int_{\Omega} \Delta P \nabla \Delta \bar{P} W d x\right| \\
& \leq 2\|\nabla W\|_{\infty}\|\nabla P\|\|\nabla \Delta P\|+2\|W\|_{\infty}\|\Delta P\|\|\nabla \Delta P\| \\
& \leq \frac{1}{3}\|\nabla \Delta P\|^{2}+C\|\nabla W\|_{H^{2}}^{\frac{n}{2}}\|\nabla W\|^{\frac{4-n}{2}}+C_{11}\|\Delta P\|^{2} \\
& \leq \frac{1}{3}\|\nabla \Delta P\|^{2}+C_{11}\|\Delta P\|^{2}+C_{12}\|\nabla \Delta W\|^{2}+C .
\end{aligned}
$$

In the same way, we can handle these terms as follows:

$$
\begin{aligned}
& \quad\left|2\left\|\Delta^{2} W\right\|^{2}-2 \operatorname{Re} \int_{\Omega} r_{1} P \Delta^{2} \bar{P} \nabla W d x-2 \operatorname{Re} \int_{\Omega} g r_{2} P \Delta^{2} \bar{P} \nabla \Delta W d x\right| \\
& \leq 2\left\|\Delta^{2} W\right\|^{2}+\left|2 \operatorname{Re} \int_{\Omega} r_{1}(P \nabla \Delta \bar{P} \Delta W+\nabla P \nabla \Delta \bar{P} \nabla W) d x\right| \\
& \quad+\left|2 \operatorname{Re} \int_{\Omega} g r_{2}\left(P \nabla \Delta \bar{P} \Delta^{2} W+\nabla P \nabla \Delta \bar{P} \nabla \Delta W\right) d x\right| \\
& \leq 2\left\|\Delta^{2} W\right\|^{2}+2\left|r_{1}\right|\|P\|\|\nabla \Delta P\|\|\Delta W\|_{\infty} \\
& \quad+2\left|r_{1}\right|\|\nabla P\|\|\nabla \Delta P\|\|\nabla W\|_{\infty}+2 g\left|r_{2}\right|\|P\|\|\nabla \Delta P\|\left\|\Delta^{2} W\right\|_{\infty} \\
& \quad+2 g\left|r_{2}\right|\|\nabla P\|\|\nabla \Delta P\|\|\nabla \Delta W\|_{\infty} \\
& \leq 2\left\|\Delta^{2} W\right\|^{2}+\frac{1}{3}\|\nabla \Delta P\|^{2}+C\|\nabla W\|_{H^{3}}^{\frac{2+n}{3}}\|\nabla W\|^{\frac{4-n}{3}} \\
& \quad+C\|\nabla W\|_{H^{2}}^{\frac{n}{2}}\|\nabla W\|^{\frac{4-n}{2}}+C\|\nabla W\|_{H^{5}}^{\frac{6+n}{5}}\|\nabla W\|^{\frac{4-n}{5}}+C\|\nabla W\|_{H^{4}}^{\frac{4+n}{4}}\|\nabla W\|^{\frac{4-n}{4}} \\
& \leq \frac{1}{3}\|\nabla \Delta P\|^{2}+g\left\|\nabla \Delta^{2} W\right\|^{2}+\frac{\delta}{4}\left\|\Delta^{3} W\right\|^{2}+4\left\|\Delta^{2} W\right\|^{2}+C\|\nabla \Delta W\|^{2}+C \\
& \leq \frac{1}{3}\|\nabla \Delta P\|^{2}+g\left\|\nabla \Delta^{2} W\right\|^{2}+\frac{\delta}{4}\left\|\Delta^{3} W\right\|^{2}+C\|\nabla W\|_{H^{4}}^{\frac{3}{2}}\|\nabla W\|^{\frac{1}{2}}+C\|\nabla \Delta W\|^{2}+C
\end{aligned}
$$




$$
\leq \frac{1}{3}\|\nabla \Delta P\|^{2}+g\left\|\nabla \Delta^{2} W\right\|^{2}+\frac{\delta}{2}\left\|\Delta^{3} W\right\|^{2}+C_{13}\|\nabla \Delta W\|^{2}+C .
$$

For the last two terms, we also have that

$$
\begin{aligned}
& \left|2 \int_{\Omega} W \nabla W \Delta^{3} W d x+2 \eta \int_{\Omega} \nabla\left(|P|^{2}\right) \Delta^{3} W d x\right| \\
\leq & 2\|W\|_{L^{\infty}}\|\nabla W\|\left\|\Delta^{3} W\right\|+4 \eta\|P\|_{L^{\infty}}\|\nabla P\|\left\|\Delta^{3} W\right\| \\
\leq & \frac{\delta}{2}\left\|\Delta^{3} W\right\|^{2}+C\|P\|_{H^{2}}^{\frac{n}{2}}\|P\|^{\frac{4-n}{2}} \\
\leq & \frac{\delta}{2}\left\|\Delta^{3} W\right\|^{2}+C_{14}\|\Delta P\|^{2}+C .
\end{aligned}
$$

Then combining (3.34)-(3.38) and noticing that $|\xi|=1$, there holds

$$
\begin{aligned}
& \frac{d}{d t}\left(\|\Delta P\|^{2}+\|\nabla \Delta W\|^{2}\right)+\|\nabla \Delta P\|^{2}+g\left\|\nabla \Delta^{2} W\right\|^{2}+\delta\left\|\Delta^{3} W\right\|^{2} \\
\leq & \left(2+C_{10}+C_{11}+C_{14}\right)\|\Delta P\|^{2}+\left(C_{12}+C_{13}\right)\|\nabla \Delta W\|^{2}+C \\
\leq & K_{3}\left(\|\Delta P\|^{2}+\|\nabla \Delta W\|^{2}\right)+C,
\end{aligned}
$$

where $K_{3}=\max \left(2+C_{10}+C_{11}+C_{14}, C_{12}+C_{13}\right)$ is a positive constant. Applying Gronwall's inequality, we have

$$
\|\Delta P\|^{2}+\|\nabla \Delta W\|^{2} \leq e^{K_{3} t}\left(\left\|\Delta P_{0}\right\|^{2}+\left\|\nabla \Delta W_{0}\right\|^{2}+C t\right),
$$

where $K_{3}$ and $C$ are positive constants. Noticing the transformation (3.2), the proof of Lemma 3.4 is complete.

Corollary 3.5. Under the conditions of Lemma 3.4, we have the estimates

$$
\|P\|_{L^{\infty}} \leq C, \quad\|\Delta Q\|_{L^{\infty}} \leq C,
$$

where $C$ are positive constants.

Proof. Based on the results of Lemma 3.1-Lemma 3.4 and the GagliardoNirenberg inequality, one can obtain this corollary easily.

Lemma 3.6. Under the conditions of Lemma 3.4, then, for the solutions of the problem (1.1)-(1.4), we have

$$
\|P\|_{H_{p e r}^{2}} \leq C, \quad\|Q\|_{H_{p e r}^{4}} \leq C,
$$

where $C$ is a positive constant.

Proof. From the estimates in Lemma 3.1-Lemma 3.4, we see $\|P\|_{H_{p e r}^{2}} \leq C$. However, we need to estimate $\|Q\|$ for $\|Q\|_{H_{p e r}^{4}}$. Considering the original equation (1.2), multiplying this equation by $Q$ and integrating over $\Omega$, we have

$$
\frac{1}{2} \frac{d}{d t}\|Q\|^{2}=\|\nabla Q\|^{2}-g\|\Delta Q\|^{2}-\delta\|\nabla \Delta Q\|^{2}-\frac{1}{2} \int_{\Omega}|\nabla Q|^{2} Q d x-\eta \int_{\Omega}|P|^{2} Q d x .
$$

By the previous lemmas and the corollary (3.30), we get

$$
\left.\left|\|\nabla Q\|^{2}-\frac{1}{2} \int_{\Omega}\right| \nabla Q\right|^{2} Q d x-\eta \int_{\Omega}|P|^{2} Q d x \mid
$$




$$
\begin{aligned}
& \leq C\|Q\|_{H^{2}}\|Q\|+\frac{1}{2}\|\nabla Q\|_{L^{\infty}}\|\nabla Q\|\|Q\|+\eta\|P\|_{L^{\infty}}\|P\|\|Q\| \\
& \leq C\|Q\|^{2}+C .
\end{aligned}
$$

Substituting (3.44) into (3.43), we find that

$$
\frac{d}{d t}\|Q\|^{2} \leq 2 C\|Q\|^{2}+C .
$$

By Gronwall's inequality and Lemma 3.1-Lemma 3.4, we get $\|Q\|_{H_{p e r}^{4}} \leq C$.

LEMma 3.7. Assume that $P_{0}(x) \in H_{\text {per }}^{3}(\Omega), Q_{0}(x) \in H_{p e r}^{5}(\Omega)$, and the conditions of Lemma 3.4. Then for the solutions of the problem (1.1)-(1.4), we have the following estimates:

$$
\|\nabla \Delta P\|^{2}+\left\|\nabla \Delta^{2} Q\right\|^{2} \leq e^{K_{4} t}\left(\left\|\nabla \Delta P_{0}\right\|^{2}+\left\|\nabla \Delta^{2} Q_{0}\right\|^{2}+C t\right)
$$

where $K_{4}$ and $C$ are positive constants.

Proof. Using the transformed equations (3.3)(3.4) as before. Taking the inner product of (3.3) with $\left(-\Delta^{3} \bar{P}\right)$ over $\Omega$ and taking the real part, we can obtain

$$
\begin{aligned}
\frac{1}{2} \frac{d}{d t}\|\nabla \Delta P\|^{2}=\xi \| & \nabla \Delta P\left\|^{2}-\right\| \Delta^{2} P \|^{2}+\operatorname{Re} \int_{\Omega}(1+i \nu)|P|^{2} P \Delta^{3} \bar{P} d x \\
+ & \operatorname{Re} \int_{\Omega} \nabla P \Delta^{3} \bar{P} W d x+\operatorname{Re} \int_{\Omega} r_{1} P \Delta^{3} \bar{P} \nabla W d x \\
& +\operatorname{Re} \int_{\Omega} g r_{2} P \Delta^{3} \bar{P} \nabla \Delta W d x
\end{aligned}
$$

Taking the inner product of (3.4) with $\Delta^{4} W$ over $\Omega$, we get

$$
\begin{aligned}
\frac{1}{2} \frac{d}{d t}\left\|\Delta^{2} W\right\|^{2}= & \left\|\nabla \Delta^{2} W\right\|^{2}-g\left\|\Delta^{3} W\right\|^{2}-\delta\left\|\nabla \Delta^{3} W\right\|^{2} \\
& -\int_{\Omega} W \nabla W \Delta^{4} W d x-\eta \int_{\Omega} \nabla\left(|P|^{2}\right) \Delta^{4} W d x .
\end{aligned}
$$

Adding the above two equalities gives

$$
\begin{aligned}
\frac{d}{d t}\left(\|\nabla \Delta P\|^{2}+\left\|\Delta^{2} W\right\|^{2}\right)=2 \xi & \|\nabla \Delta P\|^{2}-2\left\|\Delta^{2} P\right\|^{2}+2\left\|\nabla \Delta^{2} W\right\|^{2}-2 g\left\|\Delta^{3} W\right\|^{2} \\
& -2 \delta\left\|\nabla \Delta^{3} W\right\|^{2}+2 \operatorname{Re} \int_{\Omega}(1+i \nu)|P|^{2} P \Delta^{3} \bar{P} d x \\
& +2 \operatorname{Re} \int_{\Omega} \nabla P \Delta^{3} \bar{P} W d x+2 \operatorname{Re} \int_{\Omega} r_{1} P \Delta^{3} \bar{P} \nabla W d x \\
& +2 \operatorname{Re} \int_{\Omega} g r_{2} P \Delta^{3} \bar{P} \nabla \Delta W d x \\
& -2 \int_{\Omega} W \nabla W \Delta^{4} W d x-2 \eta \int_{\Omega} \nabla\left(|P|^{2}\right) \Delta^{4} W d x .
\end{aligned}
$$

Now using the estimates of previous lemmas and corollaries, we can majorize the right hand of (3.49) as follows. First, we have

$$
\left.\left|2 \operatorname{Re} \int_{\Omega}(1+i \nu)\right| P\right|^{2} P \Delta^{3} \bar{P} d x \mid
$$


1468

GLOBAL SMOOTH SOLUTIONS OF GENERALIZED KS-CGL EQUATIONS

$$
\begin{aligned}
& \left|2 \operatorname{Re} \int_{\Omega}(1+i \nu)\left(2(\nabla P)^{2} \bar{P}+2|P|^{2} \Delta P+4 P|\nabla P|^{2}+P^{2} \Delta \bar{P}\right) \Delta^{2} \bar{P} d x\right| \\
\leq & 2|1+i \nu|\left(6\|\nabla P\|_{\infty}^{2}\|P\|+3\|P\|_{\infty}^{2}\|\Delta P\|\right)\left\|\Delta^{2} P\right\| \\
\leq & \frac{1}{4}\left\|\Delta^{2} P\right\|^{2}+C\|P\|_{H^{3}}^{\frac{2+n}{3}}\|P\|^{\frac{4-n}{3}}+C \\
\leq & \frac{1}{4}\left\|\Delta^{2} P\right\|^{2}+C_{14}\|\nabla \Delta P\|^{2}+C \\
& \left|2 \operatorname{Re} \int_{\Omega} \nabla P \Delta^{3} \bar{P} W d x\right| \\
& =\left|2 \operatorname{Re} \int_{\Omega}(\Delta W \nabla P+2 \nabla W \Delta P+W \nabla \Delta P) \Delta^{2} \bar{P} d x\right| \\
& \leq 2\left(\|\Delta W\|\|\nabla P\|_{\infty}+2\|\nabla W\|_{\infty}\|\Delta P\|+\|W\|_{\infty}\|\nabla \Delta P\|\right)\left\|\Delta^{2} P\right\| \\
& \leq \frac{1}{4}\left\|\Delta^{2} P\right\|^{2}+C\|\nabla P\|_{H^{2}}^{\frac{n}{2}}\|\nabla P\|^{\frac{4-n}{2}}+C\|\nabla \Delta P\|^{2}+C \\
& \leq \frac{1}{4}\left\|\Delta^{2} P\right\|^{2}+C_{15}\|\nabla \Delta P\|^{2}+C,
\end{aligned}
$$

and

$$
\begin{aligned}
& \left|2 \operatorname{Re} \int_{\Omega} r_{1} P \Delta^{3} \bar{P} \nabla W d x\right| \\
= & \left|2 \operatorname{Re} \int_{\Omega}(\Delta P \nabla W+2 \nabla P \Delta W+P \nabla \Delta W) \Delta^{2} \bar{P} d x\right| \\
\leq & 2\left(\|\nabla W\|_{\infty}\|\Delta P\|+2\|\Delta W\|\|\nabla P\|_{\infty}+\|P\|_{\infty}\|\nabla \Delta W\|\right)\left\|\Delta^{2} P\right\| \\
\leq & \frac{1}{4}\left\|\Delta^{2} P\right\|^{2}+C\|\nabla P\|_{H^{2}}^{\frac{n}{2}}\|\nabla P\|^{\frac{4-n}{2}}+C \\
\leq & \frac{1}{4}\left\|\Delta^{2} P\right\|^{2}+C_{16}\|\nabla \Delta P\|^{2}+C .
\end{aligned}
$$

Similarly, use the Gagliardo-Nirenberg inequality and previous estimates to obtain that

$$
\begin{aligned}
& \left|2\left\|\nabla \Delta^{2} W\right\|^{2}+2 \operatorname{Re} \int_{\Omega} g r_{2} P \Delta^{3} \bar{P} \nabla \Delta W d x\right| \\
\leq & 2\left\|\nabla \Delta^{2} W\right\|^{2}+\left|2 \operatorname{Re} \int_{\Omega} g r_{2}\left(\nabla \Delta^{2} W P+2 \Delta^{2} W \nabla P+\nabla \Delta W \Delta P\right) \Delta^{2} \bar{P} d x\right| \\
\leq & 2\left\|\nabla \Delta^{2} W\right\|^{2}+2 g\left|r_{2}\right|\left(\|P\|_{\infty}\left\|\nabla \Delta^{2} W\right\|+2\|\nabla P\|\left\|\Delta^{2} W\right\|_{\infty}\right)\left\|\Delta^{2} P\right\| \\
& \quad+2 g\left|r_{2}\right|\|\Delta P\|\|\nabla \Delta W\|_{\infty}\left\|\Delta^{2} P\right\| \\
\leq & \frac{1}{4}\left\|\Delta^{2} P\right\|^{2}+2\left\|\nabla \Delta^{2} W\right\|^{2}+C\|W\|_{H^{6}}^{\frac{5}{3}}\|W\|^{\frac{2}{3}} \\
& \quad+C\|\Delta W\|_{H^{4}}^{\frac{4+n}{3}}\|\Delta W\|^{\frac{4-n}{4}}+C\|\nabla W\|_{H^{4}}^{\frac{4+n}{3}}\|\nabla W\|^{\frac{4-n}{4}} \\
\leq & \frac{1}{4}\left\|\Delta^{2} P\right\|^{2}+\frac{g}{2}\left\|\Delta^{3} W\right\|^{2}+4\left\|\nabla \Delta^{2} W\right\|^{2}+C\left\|\Delta^{2} W\right\|^{2}+C \\
\leq & \frac{1}{4}\left\|\Delta^{2} P\right\|^{2}+g\left\|\Delta^{3} W\right\|^{2}+C_{17}\left\|\Delta^{2} W\right\|^{2}+C .
\end{aligned}
$$

For the last two terms, we get

$$
\left|-2 \int_{\Omega} W \nabla W \Delta^{4} W d x-2 \eta \int_{\Omega} \nabla\left(|P|^{2}\right) \Delta^{4} W d x\right|
$$




$$
\begin{aligned}
\leq & \left|2 \int_{\Omega}\left((\nabla W)^{2}+W \Delta W\right) \nabla \Delta^{3} W d x\right|+2 \eta\left|\int_{\Omega}\left(\Delta P \bar{P}+2|\nabla P|^{2}+P \Delta \bar{P}\right) \nabla \Delta^{3} W d x\right| \\
\leq & 2\left(\|\nabla W\|_{\infty}\|\nabla W\|+\|W\|_{\infty}\|\Delta W\|\right)\left\|\nabla \Delta^{3} W\right\| \\
& \quad+2 \eta\left(\|\Delta P\|\|P\|_{\infty}+2\|\nabla P\|_{\infty}\|\nabla P\|+\|P\|_{\infty}\|\Delta P\|\right)\left\|\nabla \Delta^{3} W\right\| \\
\leq & \delta\left\|\nabla \Delta^{3} W\right\|^{2}+C_{18}\|\nabla \Delta P\|^{2}+C
\end{aligned}
$$

Substituting (3.50)-(3.54) into (3.49) yields that

$$
\begin{aligned}
& \frac{d}{d t}\left(\|\nabla \Delta P\|^{2}+\left\|\Delta^{2} W\right\|^{2}\right)+\left\|\Delta^{2} P\right\|^{2}+g\left\|\Delta^{3} W\right\|^{2}+\delta\left\|\nabla \Delta^{3} W\right\|^{2} \\
\leq & \left(2+C_{14}+C_{15}+C_{16}+C_{18}\right)\|\nabla \Delta P\|^{2}+C_{17}\left\|\Delta^{2} W\right\|^{2}+C \\
\leq & K_{4}\left(\|\nabla \Delta P\|^{2}+\left\|\Delta^{2} W\right\|^{2}\right)+C,
\end{aligned}
$$

where $K_{4}=\max \left(2+C_{14}+C_{15}+C_{16}+C_{18}, C_{17}\right)$ is a positive constant. Applying Gronwall's inequality and transformation (3.2) completes the proof of Lemma 3.7.

Corollary 3.8. Under the conditions of Lemma 3.7, we have the estimates

$$
\|\nabla P\|_{L^{\infty}} \leq C, \quad\|\nabla \Delta Q\|_{L^{\infty}} \leq C,
$$

for the solutions of the problem (1.1)-(1.4), where $C$ is a positive constant.

Lemma 3.9. Under the conditions of Lemma 3.1-Lemma 3.7, we have the estimate

$$
\left\|P_{t}\right\|^{2}+\left\|Q_{t}\right\|^{2}+\left\|\nabla Q_{t}\right\|^{2} \leq C
$$

for the solutions of the problem (1.1)-(1.4), where $C$ is a positive constant.

Proof. We differentiate equation (1.1) and (1.2) with respect to $t$ once, take the inner product of the resulting equations with $\bar{P}_{t}$ and $\left(Q_{t}-\Delta Q_{t}\right)$ respectively, and take the real parts to obtain

$$
\begin{gathered}
\frac{1}{2} \frac{d}{d t}\left\|P_{t}\right\|^{2}=\xi\left\|P_{t}\right\|^{2}-\left\|\nabla P_{t}\right\|^{2}-\operatorname{Re} \int_{\Omega}(1+i \nu)\left(2|P|^{2}\left|P_{t}\right|^{2}+P^{2} \bar{P}_{t}^{2}\right) d x \\
-\operatorname{Re} \int_{\Omega}\left(\nabla P_{t} \nabla Q+\nabla P \nabla Q_{t}\right) \bar{P}_{t} d x \\
-\operatorname{Re} \int_{\Omega} r_{1}\left(\left|P_{t}\right|^{2} \Delta Q+P \Delta Q_{t} \bar{P}_{t}\right) d x \\
-\operatorname{Re} \int_{\Omega} g r_{2}\left(\left|P_{t}\right|^{2} \Delta^{2} Q+P \Delta^{2} Q_{t} \bar{P}_{t}\right) d x
\end{gathered}
$$

and

$$
\begin{aligned}
\frac{1}{2} \frac{d}{d t}\left(\left\|Q_{t}\right\|^{2}+\left\|\nabla Q_{t}\right\|^{2}\right)=\| & \nabla Q_{t}\left\|^{2}-(g-1)\right\| \Delta Q_{t}\left\|^{2}-(\delta+g)\right\| \nabla \Delta Q_{t} \|^{2} \\
& -\delta\left\|\Delta^{2} Q_{t}\right\|^{2}-\int_{\Omega} \nabla Q \nabla Q_{t}\left(Q_{t}-\Delta Q_{t}\right) d x \\
& -\eta \int_{\Omega}\left(P_{t} \bar{P}+P \bar{P}_{t}\right)\left(Q_{t}-\Delta Q_{t}\right) d x
\end{aligned}
$$


Adding (3.58) and (3.59) together, noticing that $|\xi|=1$, and using the estimates in Lemma 3.1-Lemma 3.7 yields

$$
\begin{aligned}
& \frac{d}{d t}\left(\left\|P_{t}\right\|^{2}+\left\|Q_{t}\right\|^{2}+\left\|\nabla Q_{t}\right\|^{2}\right) \\
& =2 \xi\left\|P_{t}\right\|^{2}-\left\|\nabla P_{t}\right\|^{2}+2\left\|\nabla Q_{t}\right\|^{2}-2(g-1)\left\|\Delta Q_{t}\right\|^{2}-2(\delta+g)\left\|\nabla \Delta Q_{t}\right\|^{2} \\
& \quad-2 \delta\left\|\Delta^{2} Q_{t}\right\|^{2}-2 \operatorname{Re} \int_{\Omega}(1+i \nu)\left(2|P|^{2}\left|P_{t}\right|^{2}+P^{2} \bar{P}_{t}^{2}\right) d x \\
& \quad-2 \operatorname{Re} \int_{\Omega}\left(\nabla P_{t} \nabla Q+\nabla P \nabla Q_{t}\right) \bar{P}_{t} d x \\
& \quad-2 \operatorname{Re} \int_{\Omega} r_{1}\left(\left|P_{t}\right|^{2} \Delta Q+P \Delta Q_{t} \bar{P}_{t}\right) d x-2 \operatorname{Re} \int_{\Omega} g r_{2}\left(\left|P_{t}\right|^{2} \Delta^{2} Q+P \Delta^{2} Q_{t} \bar{P}_{t}\right) d x \\
& \quad-2 \int_{\Omega} \nabla Q \nabla Q_{t}\left(Q_{t}-\Delta Q_{t}\right) d x-2 \eta \int_{\Omega}\left(P_{t} \bar{P}+P \bar{P}_{t}\right)\left(Q_{t}-\Delta Q_{t}\right) d x \\
& \leq 2\left\|P_{t}\right\|^{2}-\left\|\nabla P_{t}\right\|^{2}+2\left\|\nabla Q_{t}\right\|^{2}+2|g-1|\left\|\Delta Q_{t}\right\|^{2}-2 \delta\left\|\nabla \Delta Q_{t}\right\|^{2} \\
& \quad-2 \delta\left\|\Delta^{2} Q_{t}\right\|^{2}+6|1+i \nu|\|P\|_{\infty}^{2}\left\|P_{t}\right\|^{2}+2\left(\|\nabla Q\|_{\infty}\left\|\nabla P_{t}\right\|+\|\nabla P\|_{\infty}\left\|\nabla Q_{t}\right\|\right)\left\|P_{t}\right\| \\
& \quad+2 r_{1 r}\|\Delta Q\|_{\infty}\left\|P_{t}\right\|^{2}+2\left|r_{1}\right|\|P\|_{\infty}\left\|\Delta Q_{t}\right\|\left\|P_{t}\right\| \\
& \quad+4 g\left|r_{2}\right|\|\nabla \Delta Q\|_{\infty}\left\|P_{t}\right\|\left\|\nabla P_{t}\right\|+2 g\left|r_{2}\right|\|\nabla P\|_{\infty}\left\|\nabla \Delta Q_{t}\right\|\left\|P_{t}\right\| \\
& \quad+2 g\left|r_{2}\right|\|P\|_{\infty}\left\|\nabla \Delta Q_{t}\right\|\left\|\nabla P_{t}\right\| \\
& \quad+2\|\nabla Q\|_{\infty}\left\|Q_{t}\right\|\left(\left\|\nabla Q_{t}\right\|+\left\|\Delta Q_{t}\right\|\right)+4 \eta\|P\|_{\infty}\left\|P_{t}\right\|\left(\left\|\nabla Q_{t}\right\|+\left\|\Delta Q_{t}\right\|\right) \\
& \leq C
\end{aligned}
$$

where we apply Young's inequality with $\varepsilon$ and the Gagliardo-Nirenberg inequality repeatedly. Thus, Gronwall's inequality yields the estimates of Lemma 3.9.

Generally based on the results of the previous lemmas and mathematical deduction, we have the following lemma for problem (1.1)-(1.4).

LEMma 3.10. Assume that $P_{0}(x) \in H^{k}(\Omega), Q_{0}(x) \in H^{k+2}(\Omega)(k \geq 3)$, and $2 \delta-$ $g^{2} r_{2 r}^{2}>0$. If $n=2$, we also suppose $|\nu| \leq \sqrt{3}$. Then for the solutions of the problem (1.1)-(1.4), we have the following estimates:

$$
\left\|\nabla^{k} P\right\|^{2}+\left\|\nabla^{k+2} Q\right\|^{2} \leq C .
$$

Furthermore, there also holds

$$
\left\|\nabla^{k-3} P_{t}\right\|^{2}+\left\|\nabla^{k-2} Q_{t}\right\|^{2} \leq C
$$

where the positive constant $C$ depends on $\left\|\nabla^{k} P_{0}\right\|$ and $\left\|\nabla^{k+2} Q_{0}\right\|$ and is independent of the period $L$.

\section{The local solutions and global solutions}

In this section, we will obtain the existence and uniqueness of the local solutions and global solutions for the periodic initial value problem (1.1)-(1.4). First, we adopt the Galerkin method to construct the approximate solutions for the problem (1.1)(1.4). Let $\omega_{j}(x)(j=1,2, \cdots)$ be the unit eigenfunctions satisfying the equation

$$
\Delta \omega_{j}+\lambda_{j} \omega_{j}=0, \quad j=1,2, \cdots, \quad \omega_{j} \in H_{0}^{1}(\Omega) \cap L^{4}(\Omega),
$$


with periodicity $\omega_{j}(x)=\omega_{j}\left(x+L e_{i}\right)(i=1,2)$ and where $\lambda_{j}(j=1,2, \cdots)$ are the distinct eigenvalues corresponding to the orthogonal basis $\left\{\omega_{j}(x)\right\}$ of $L^{2}(\Omega)$. Thus the approximate solutions can be written as

$$
P_{m}(x, t)=\sum_{j=1}^{m} \alpha_{j m}(t) \omega_{j}(x), \quad Q_{m}(x, t)=\sum_{j=1}^{m} \beta_{j m}(t) \omega_{j}(x) .
$$

According to the Galerkin method, these undetermined coefficients $\alpha_{j m}(t)$ and $\beta_{j m}(t)$ have to satisfy the following initial value problem of the system of ordinary differential equations

$$
\begin{gathered}
\left(P_{m t}, \omega_{j}\right)=\xi\left(P_{m}, \omega_{j}\right)-(1+i \mu)\left(\nabla P_{m}, \nabla \omega_{j}\right)-(1+i \nu)\left(\left|P_{m}\right|^{2} P_{m}, \omega_{j}\right) \\
-\left(\nabla P_{m} \nabla Q_{m}, \omega_{j}\right)-r_{1}\left(P_{m} \Delta Q_{m}, \omega_{j}\right)-g r_{2}\left(P_{m} \Delta^{2} Q_{m}, \omega_{j}\right), \\
\left(Q_{m t}, \omega_{j}\right)=\left(\nabla Q_{m}, \nabla \omega_{j}\right)-g\left(\Delta Q_{m}, \Delta \omega_{j}\right)-\delta\left(\nabla \Delta Q_{m}, \nabla \Delta \omega_{j}\right) \\
-\frac{1}{2}\left(\left|\nabla Q_{m}\right|^{2}, \omega_{j}\right)-\eta\left(\left|P_{m}\right|^{2}, \omega_{j}\right),
\end{gathered}
$$

with initial conditions

$$
P_{m}(x, 0)=P_{0 m}(x), \quad Q_{m}(x, 0)=Q_{0 m}(x),
$$

where $0 \leq t \leq T$ and $j=1,2, \ldots, m$.

We assume that

$$
P_{0 m}(x) \stackrel{H_{p e r}^{3}(\Omega)}{\longrightarrow} P_{0}(x), \quad Q_{0 m}(x) \stackrel{H_{p e r}^{5}(\Omega)}{\longrightarrow} Q_{0}(x), \quad m \rightarrow \infty .
$$

As in the proof of Lemma 3.1-Lemma 3.9, we can establish estimates of the solutions of the problem (1.1)-(1.4) which are uniform for $m$. By using the compact principle, we can prove the following result.

TheOrem 4.1 (Local existence). Assume that $P_{0}(x) \in H_{\text {per }}^{3}(\Omega), Q_{0}(x) \in$ $H_{p e r}^{5}(\Omega)$, and $2 \delta-g^{2} r_{2 r}^{2}>0$. If $n=2$, we also suppose $|\nu| \leq \sqrt{3}$. Then the periodic initial value problem (1.1)-(1.4) possesses periodic local solutions $P(x, t)$ and $Q(x, t)$, which satisfy

$$
\begin{aligned}
& P(x, t) \in L^{\infty}\left(0, t_{0} ; H_{p e r}^{3}(\Omega)\right), \quad P_{t}(x, t) \in L^{\infty}\left(0, t_{0} ; L_{p e r}^{2}(\Omega)\right), \\
& Q(x, t) \in L^{\infty}\left(0, t_{0} ; H_{p e r}^{5}(\Omega)\right), \quad Q_{t}(x, t) \in L^{\infty}\left(0, t_{0} ; H_{p e r}^{1}(\Omega)\right),
\end{aligned}
$$

where $t_{0}$ depends on $\left\|P_{0}(x)\right\|_{H_{p e r}^{3}}$ and $\left\|Q_{0}(x)\right\|_{H_{p e r}^{5}}$.

Theorem 4.2 (Global existence). Under the conditions of Theorem 4.1. Then there exists global solutions $P(x, t)$ and $Q(x, t)$, which satisfy

$$
\begin{gathered}
P(x, t) \in L^{\infty}\left(0, T ; H_{p e r}^{3}(\Omega)\right), \quad P_{t}(x, t) \in L^{\infty}\left(0, T ; L_{p e r}^{2}(\Omega)\right), \\
Q(x, t) \in L^{\infty}\left(0, T ; H_{p e r}^{5}(\Omega)\right), \quad Q_{t}(x, t) \in L^{\infty}\left(0, T ; H_{p e r}^{1}(\Omega)\right),
\end{gathered}
$$

for the periodic initial value problem (1.1)-(1.4). 
Proof. From Theorem 4.1 we know that the local solutions for the problem (1.1)-(1.4) exist and $t_{0}$ depends on $\left\|P_{0}(x)\right\|_{H_{p e r}^{3}}$ and $\left\|Q_{0}(x)\right\|_{H_{p e r}^{5}}$. According to the a priori estimates in Section 3 and by the so-called continuity method, we can obtain the global solutions for the problem (1.1)-(1.4) easily.

Theorem 4.3 (Uniqueness for global solutions). Under the conditions of Theorem 4.2, the global solutions $P(x, t)$ and $Q(x, t)$ of the periodic initial value problem (1.1)-(1.4) are unique.

Proof. If $P_{1}(x, t), Q_{1}(x, t)$ and $P_{2}(x, t), Q_{2}(x, t)$ are two solutions of problem (1.1)-(1.4), then the differences $P=P_{1}(x, t)-P_{2}(x, t), Q(x, t)=Q_{1}(x, t)-Q_{2}(x, t)$ will satisfy

$$
\begin{gathered}
P_{t}=\xi P+(1+i \mu) \Delta P-(1+i \nu)\left(\left|P_{1}\right|^{2} P_{1}-\left|P_{2}\right|^{2} P_{2}\right)-\left(\nabla P_{1} \nabla Q_{1}-\nabla P_{2} \nabla Q_{2}\right) \\
-r_{1}\left(P_{1} \Delta Q_{1}-P_{2} \Delta Q_{2}\right)-g r_{2}\left(P_{1} \Delta^{2} Q_{1}-P_{2} \Delta^{2} Q_{2}\right), \\
Q_{t}=-\Delta Q-g \Delta^{2} Q+\delta \Delta^{3} Q-\frac{1}{2}\left(\left|\nabla Q_{1}\right|^{2}-\left|\nabla Q_{2}\right|^{2}\right)-\eta\left(\left|P_{1}\right|^{2}-\left|P_{2}\right|^{2}\right), \\
P\left(x+L e_{i}, t\right)=P(x, t), \quad Q\left(x+L e_{i}, t\right)=Q(x, t), \\
P(x, 0)=0, \quad Q(x, 0)=0 .
\end{gathered}
$$

Taking the inner product of (4.7) with $\bar{P}$ and taking the real parts, taking the inner product of (4.8) with $-\Delta Q$ over $\Omega$, then adding these two equations together, we obtain

$$
\begin{aligned}
& \frac{d}{d t}\left(\|P\|^{2}+\|\nabla Q\|^{2}\right) \\
&=2\|P\|^{2}-2\|\nabla P\|^{2}+2\|\Delta Q\|^{2}-2 g\|\nabla \Delta Q\|^{2}-2 \delta\left\|\Delta^{2} Q\right\|^{2} \\
& \quad-\operatorname{Re} \int_{\Omega} 2(1+i \nu)\left(\left|P_{1}\right|^{2} P_{1}-\left|P_{2}\right|^{2} P_{2}\right) \bar{P} d x-2 \operatorname{Re} \int_{\Omega}\left(\nabla P_{1} \nabla Q_{1}-\nabla P_{2} \nabla Q_{2}\right) \bar{P} d x \\
&-2 \operatorname{Re} \int_{\Omega} r_{1}\left(P_{1} \Delta Q_{1}-P_{2} \Delta Q_{2}\right) \bar{P} d x-2 \operatorname{Re} \int_{\Omega} g r_{2}\left(P_{1} \Delta^{2} Q_{1}-P_{2} \Delta^{2} Q_{2}\right) \bar{P} d x \\
&+\int_{\Omega}\left(\left|\nabla Q_{1}\right|^{2}-\left|\nabla Q_{2}\right|^{2}\right) \Delta Q d x+2 \eta \int_{\Omega}\left(\left|P_{1}\right|^{2}-\left|P_{2}\right|^{2}\right) \Delta Q d x \\
& \leq C\left(\|P\|^{2}+\|\nabla Q\|^{2}\right),
\end{aligned}
$$

where we majorize the right-hand side of (4.11) with Young's inequality with $\varepsilon$ and the Gagliardo-Nirenberg inequality, because $P_{1}(x, t), Q_{1}(x, t)$ and $P_{2}(x, t), Q_{2}(x, t)$ are the solutions of the problem (1.1)-(1.4) satisfying the estimates in Lemma 3.1-Lemma 3.9 .

By Gronwall's inequality and noticing the conditions (4.10), we can complete the proof of the Theorem 4.3.

More generally, we have the following existence and uniqueness theorems for the global smooth solutions from Lemma 3.10.

THEOREM 4.4 (Existence and uniqueness for global smooth solutions). Suppose that $P_{0}(x) \in H_{\text {per }}^{k}(\Omega), Q_{0}(x) \in H_{\text {per }}^{k+2}(\Omega)(k \geq 3)$ and $2 \delta-g^{2} r_{2 r}^{2}>0$. If $n=2$, we also 
assume $|\nu| \leq \sqrt{3}$. Then there exists unique global smooth solutions $P(x, t)$ and $Q(x, t)$, which satisfy

$$
\begin{gathered}
P(x, t) \in L^{\infty}\left(0, T ; H_{p e r}^{k}(\Omega)\right), \quad P_{t}(x, t) \in L^{\infty}\left(0, T ; H_{p e r}^{k-3}(\Omega)\right), \\
Q(x, t) \in L^{\infty}\left(0, T ; H_{p e r}^{k+2}(\Omega)\right), \quad Q_{t}(x, t) \in L^{\infty}\left(0, T ; H_{p e r}^{k-2}(\Omega)\right),
\end{gathered}
$$

for the periodic initial value problem (1.1)-(1.4).

\section{Cauchy problem}

In previous sections, we studied the existence and uniqueness of the global smooth solutions for the periodic initial value problem (1.1)-(1.4). In this section, we will discuss the Cauchy problem (1.1)(1.2)(1.5) in the infinite domain $\Omega_{T}=\left\{(x, t) \mid x \in \mathbb{R}^{n}, 0 \leq\right.$ $t \leq T\}(n=1,2)$. Because we have supposed that $P(x, t), Q(x, t)$ and some of their derivatives with respect to $x$ tend to zero as $|x| \rightarrow \infty$, then the a priori estimates in Section 3 also hold for the solutions of the problem (1.1)(1.2)(1.5) . Furthermore, the a priori estimates are bounded and independent of the period $L$ of the domain $\Omega$, thus we can choose sequence $L_{k}\left(k \rightarrow \infty, L_{k} \rightarrow \infty\right)$ and obtain global existence in $\left[0, T_{k}\right]$. Then we can employ the usual method of limiting process for $L_{k} \rightarrow \infty(k \rightarrow \infty)$, which is the so-called diagonal selection, to obtain the solutions of Cauchy problem. The global existence and uniqueness theorems for the Cauchy problem (1.1)(1.2)(1.5) which are parallel to Theorem 4.2, Theorem 4.3, and Theorem 4.4 can be stated as follows.

THEOREM 5.1. Suppose that $2 \delta-g^{2} r_{2 r}^{2}>0$, and if $n=2$, we also assume $|\nu| \leq \sqrt{3}$. If $P_{0}(x) \in H^{3}\left(\mathbb{R}^{n}\right), Q_{0}(x) \in H^{5}\left(\mathbb{R}^{n}\right)$, then there exists global solutions

$$
\begin{gathered}
P(x, t) \in L^{\infty}\left(0, T ; H^{3}\left(\mathbb{R}^{n}\right)\right), \quad P_{t}(x, t) \in L^{\infty}\left(0, T ; L^{2}\left(\mathbb{R}^{n}\right)\right), \\
Q(x, t) \in L^{\infty}\left(0, T ; H^{5}\left(\mathbb{R}^{n}\right)\right), \quad Q_{t}(x, t) \in L^{\infty}\left(0, T ; H^{1}\left(\mathbb{R}^{n}\right)\right),
\end{gathered}
$$

for the Cauchy problem (1.1)(1.2)(1.5).

Furthermore, if $P_{0}(x) \in H^{k}\left(\mathbb{R}^{n}\right), Q_{0}(x) \in H^{k+2}\left(\mathbb{R}^{n}\right) \quad(k \geq 4)$, then there exists unique global smooth solutions $P(x, t), Q(x, t)$ for the Cauchy problem (1.1)(1.2)(1.5), which satisfy

$$
\begin{gathered}
P(x, t) \in L^{\infty}\left(0, T ; H^{k}\left(\mathbb{R}^{n}\right)\right), \quad P_{t}(x, t) \in L^{\infty}\left(0, T ; H^{k-3}\left(\mathbb{R}^{n}\right)\right), \\
Q(x, t) \in L^{\infty}\left(0, T ; H^{k+2}\left(\mathbb{R}^{n}\right)\right), \quad Q_{t}(x, t) \in L^{\infty}\left(0, T ; H^{k-2}\left(\mathbb{R}^{n}\right)\right) .
\end{gathered}
$$

\section{REFERENCES}

[1] A.A. Golovin, B.J. Matkowsky, A. Bayliss, and A.A. Nepomnyashchy, Coupled KS-CGL and coupled Burgers-CGL equations for flames governed by a sequential reaction, Physica D, 129, 253-298, 1999.

[2] F.A. Williams, Combustion Theory, Benjamin Cummings, Menlo Park, 1985.

[3] J. Peláez and A. Lin̈án, Structure and stability of flames with two sequential reactions, SIAM J. Appl. Math., 45, 503-522, 1985.

[4] J. Peláez, Stability of premixed flames with two thin reaction layers, SIAM J. Appl. Math., 47(4), 781-799, 1987. 
[5] A.A. Nepomnyashchy, Order parameter equations for long wavelength instabilities, Physica D, 86, 90-95, 1995.

[6] G.I. Sivashinsky, Nonlinear analysis of hydrodynamic instability in laminar flames I. Derivation of basic equations, Acta Astro., 4(11-12), 1177-1206, 1977.

[7] D.L. Li, B.L. Guo, and X.H. Liu, Existence of global solution for complex Ginzburg Landau equation in three dimensions, Appl. Math. J. Chinese Univ. Ser. A, 19(4), 409-416, 2004.

[8] D.L. Li, B.L. Guo, and X.H. Liu, Regularity of the attractor for 3-D complex Ginzburg-Landau equation, Acta Math. Appl. Sin., English Series, 27(2), 289-302, 2011.

[9] J.M. Ghidaglia and B. Héron, Dimension of the attractor associated to the Ginzburg-Landau equation, Physica D: Nonlinear Phenomena, 28(3), 282-304, 1987.

[10] B.L. Guo, The existence and nonexistence of a global smooth solution for the initial value problem of generalized Kuramoto-Sivashinsky type equations, J. Math. Res. Exposition, 11(1), 57-70, 1991.

[11] B.L. Guo, The nonlinear Galerkin methods for the generalized Kuramoto-Sivashinsky type equations, Adv. Math., 22(2), 182-184, 1993.

[12] C.M. Postlethwaite and M. Silber, Spatial and temporal feedback control of traveling wave solutions of the two-dimensional complex Ginzburg-Landau equation, Physica D: Nonlinear Phenomena, 236(1), 65-74, 2007.

[13] J.A. Sherratt, M.J. Smith, and J.D.M. Rademacher, Patterns of sources and sinks in the complex Ginzburg-Landau equation with zero linear dispersion, SIAM J. Appl. Dyn. Syst., 9(3), 883-918, 2010.

[14] G.G. Doronin and N.A. Larkin, Kuramoto-Sivashinsky model for a dusty medium, Math. Meth. Appl. Sci., 26(3), 179-192, 2003.

[15] T. MacKenzie and A.J. Roberts, Accurately model the Kuramoto-Sivashinsky dynamics with holistic discretization, SIAM J. Appl. Dyn. Syst., 5(3), 365-402, 2006.

[16] A.A. Golovin, A.A. Nepomnyashchy, and B.J. Matkowsky, Traveling and spiral waves for sequential flames with translation symmetry: Coupled CGL-Burgers equations, Physica D, 160, 1-28, 2001.

[17] L.C. Evans, Partial Differential Equations, Graduate Studies in Mathematics, Providence, Rhode Island, American Mathematical Society, AMS, 19, 1988.

[18] A. Friedman, Partial Differential Equations, Holt, Reinhart and Winston, New York, 1969. 